\title{
Theranostic Applications of Magnetic Nanoparticles in Breast Cancer
}

\author{
Khan MFA', MD Ahammad F', Khan $\mathrm{NH}^{3}$ and \\ Ahmed Ni* \\ ${ }^{1}$ Department of Pharmacy, Quaid-i- Azam University \\ Islamabad, Pakistan \\ ${ }^{2}$ School of Basic Medicines, Henan University, China \\ ${ }^{3}$ School of Life Sciences, Henan University, China \\ *Corresponding author: Naveed Ahmed, Department \\ of Pharmacy, Quaid-i- Azam University Islamabad, \\ Pakistan
}

Received: July 31, 2021; Accepted: August 20, 2021; Published: August 27, 2021

\begin{abstract}
Magnetic Nanoparticles (MNPs) with theranostic applications have engrossed innumerable attention in breast cancer therapies. Breast cancer is a malignancy that affects numerous women globally and has a bad prognosis. These novel MNP formulations have ultra-small particle sizes, high intrinsic magnetic field characteristic, effective imaging, drug targeting, and drug delivery characteristics. MNP also has anticancer properties as well as imaging and magnetic targeting abilities. Theranostic application of MNP can be used in preclinical and clinical settings, and it could be beneficial in cancer treatment and imaging. Furthermore, if these nanoparticles can be functionalized with antibodies or ligands, they can be used as new platforms in a variety of biological applications. In a nutshell, theranostic application of MNP is a small step in the long fight against breast cancer.
\end{abstract}

\section{Introduction}

Breast cancer is the second largest cause of mortality among women. Breast cancer is a multi-step process involving various cell types, and prevention is still difficult around the world. One of the most effective ways to avoid breast cancer is to diagnose it early. Breast cancer patients in some developed nations have a 5-year relative survival rate of more than $80 \%$ due to early detection and treatment [1]. Breast cancer is a malignancy that affects numerous women globally but has a poor prognosis because they do not overexpress estrogen receptor, progesterone receptor, or human epidermal growth factor receptor. Majority of these tumors are referred to as triple-negative breast cancers [2]. Triple-negative breast cancer is more likely to recur than the other 2 subtypes: hormone receptor positive/ERBB2 negative (70\% of patients), ERBB2 positive (15-20\%). The survival rate for stage I triple-negative tumors is $85 \%$ to $99 \%$ compared to $94 \%$ for hormone receptor positive and ERBB2 positive tumors [3].

Early diagnosis and treatment of disease is the most significant component of a favorable prognosis. Nanoparticles (NPs) have a unique role in the field of nanomedicine because they can be used in an extensive range of applications including disease diagnosis, drug delivery and therapy. NPs are mainly selected for drug delivery applications because of their wide variety in structure, shape and nature [4]. Nano nanotechnology has created new paradigms for theranostics. Magnetic nanoparticles could be used as a diagnostic agent within a single platform. In this Account, we examine the potential advantages and opportunities afforded by magnetic nanoparticles as platform materials [5].

MNPs (mainly iron oxide nanoparticles) are frequently used in a variety of medical applications: drug nanocarriers, contrast imaging agents in magnetic resonance imaging (MRI), local hyperthermia, and magnetic targeting have all been used using MNPs [6]. The utilisation of innovative therapeutic tactics such as nanotheranostics, which use tailored diagnostic therapy, is increasing as cancer nanotechnology advances. Magnetic nanoformulation with basic imaging and medication delivery properties include photo thermal therapy, thermos sensitive chemotherapy, and visible/luminescence/ near infrared/multimodal imaging, in addition to drug delivery [7].

\section{Theramostic Applications of MNP}

By employing nanoparticles, individualized targeted theranostic nanomedicine has emerged as a viable approach for increasing sensitivity and specificity during diagnosis, as well as the possibility of survival. Theranostic NPs have the potential to be one of the most useful technologies for breast cancer detection, diagnosis, and treatment [8]. Nano-systems have been designed to combat the uncontrolled proliferation of tumor cells. In practice, MNPs-based systems including anticancer medicines have demonstrated a higher therapeutic index than traditional chemotherapy, resulting in a significant reduction in nano-system toxicity [9].

Magnetic nanoparticles have superior imaging, therapeutic, and biocompatibility capabilities when coated with polyarabic acid. This coating facilitates cell membrane penetration and internalization into breast cancer cells. It also enables for the reversible loading of the chemotherapeutic medication Doxorubicin, which reduces tumor growth in a mouse model of breast cancer [4]. Poly-L-Lysine (PLL) polymer coating on $\mathrm{Au}-\mathrm{Fe}_{3} \mathrm{O}_{4}$ nanostructure integrates both diagnostics and photo thermal ablation of malignancies. The cells inhibit the growth of BT- 474 and MDA-MB- 231 cells by 40 and $60 \%$, respectively. Theranostic agents is effective for intracellular uptake and hyper-thermal treatments of breast cancer cells [10].

MNP Hybrid Chitosan-grafted Graphene Oxide/Magnetic Nanoparticle System with doxorubicin showed improved performance against cancer cells MCF7. This system acts as $\mathrm{pH}$ sensitive smart nano-carrier to target malignant cells [11]. Gold nano-shell around PLGA nanoparticles co-loaded with Perfluorooctyl Bromide (PFOB) and Superparamagnetic Iron Oxide Nanoparticles (SPIOs) reveal receptor-mediated specific binding to Her2-positive human breast cancer SKBR3 cells. Her2-GPH NPs could serve as effective photo- 
absorbers to specifically induce SKBR3 cell death upon near-infrared laser irradiation. In a crux, Her2-GPH NPs are innovative theranostic agents with a lot of promise for facilitating early non-invasive breast cancer diagnosis and adjuvant therapy [12].

An iron oxide nanoparticle core coated with Cyclodextrin (CD) and a pluronic polymer (F68) with curcumin showed better absorption in cancer cells in a composite MNP formulation. More crucially, magnetic targeting of MNP-Curcumin allowed for enhanced Curcumin delivery to cancer cells $[2,13]$. The conjugation of antiVEGF (Vascular endothelial growth factor) antibodies with bovine serum albumin coated PEGylated magnetic nanoparticles improves binding with the murine breast cancer $4 \mathrm{~T} 1$ cell line and promotes the delivery of doxorubicin to tumor cells. Magnetic Resonance Imaging (MRI) of 4T1 tumors 24 hours after intravenous injection revealed nanoparticle accumulation in tumors, enabling for cancer therapy and diagnostics at the same time $[14,15]$.

A hybrid peptide (biotinylation site; HER2/neu-binding motif; the dockerin domain and cohesin domain) coupled with of the Quantum Dots (QDs) peptide and MNPs-conjugated peptide could detect and destroy HER2/neu-positive breast cancer cells as a result of the QDs-emitted fluorescence and the MNPs-mediated hyperthermia [16]. Therefore, Targeted nanoparticle therapy could pave the way for tumor specific biomarkers and effective strategies for non-accessible tumor regions, resulting in a tangible change in the cancer world's history [9].

\section{Future Perspectives}

Despite the fact that many features of these nano-systems have been clarified, there are still numerous concerns about nanoparticle biocompatibility. These concerns include the fact that these nanoparticles are very small in size but are utilized in hefty numbers for tumor ablation, making it difficult for biological systems to clear them from the metabolism. Moreover, a variety of neurodegenerative disorders can occur due to induction of nanoparticles which triggers a cascade of Reactive Oxygen Species (ROS) formation; thus, impairing mitochondrial function and damaging DNA. The aforementioned shortcomings of nano-systems could be overcome by using biocompatible polymers and surfactants to cross the human body's barriers; hence, augmenting the nano-systems' biocompatibility.

\section{Conclusion}

The development of nanomaterials that combine diagnostic and therapeutic functions on a single nanoplatform is critical for drug delivery. Clinical translation of breast cancer therapy requires the creation of safe, efficient, and biocompatible drug nanoformulations with theranostic characteristics. MNP improves cancer cell uptake, increases mitochondrial membrane potential loss, and produces more ROS, indicating that it could be very useful in anticancer therapies. Furthermore, improved MRI properties show that nanoformulation has good imaging qualities. In a nutshell, these findings suggest that nanoparticles can be used in tailored delivery systems for efficient image directed breast cancer therapies.

\section{Acknowledgments}

The authors gratefully acknowledge the assistance and motivation energy of Dr. Muhammad Sarfaraz to accomplish this manuscript.
Hussain pays million thanks to his Piayree Lala for his support and presence.

\section{Funding}

This work was supported by grants from the Higher Education Commission of Pakistan and Quaid I Aazm University Islamabad.

\section{References}

1. Sun YS, Zhao Z, Yang ZN, Xu F, Lu HJ, Zhu ZY, et al. Risk factors and preventions of breast cancer. Int J Biol Sci. 2017; 13: 1387-1397.

2. Yallapu MM, Othman SF, Curtis ET, Bauer NA, Chauhan N, Kumar D, et al. Curcumin-loaded magnetic nanoparticles for breast cancer therapeutics and imaging applications. Int J Nanomedicine. 2012; 7: 1761-1779.

3. Waks AG, Winer EP. Breast Cancer Treatment: A Review. JAMA - J Am Med Assoc. 2019; 321: 288-300.

4. Patitsa M, Karathanou K, Kanaki Z, Tzioga L, Pippa N, Demetzos C, et al. Magnetic nanoparticles coated with polyarabic acid demonstrate enhanced drug delivery and imaging properties for cancer theranostic applications. Sci Rep. 2017; 7: 1-8.

5. Yoo D, Lee JH, Shin $\mathrm{TH}$, Cheon J. Theranostic magnetic nanoparticles. Acc Chem Res. 2011; 44: 863-874.

6. Gloag L, Mehdipour M, Chen D, Tilley RD, Gooding JJ. Advances in the Application of Magnetic Nanoparticles for Sensing. Adv Mater. 2019; 31: $1-26$.

7. Cardoso VF, Francesko A, Ribeiro C, Bañobre-López M, Martins P, LancerosMendez S. Advances in Magnetic Nanoparticles for Biomedical Applications. Adv Healthc Mater. 2018; 7: 1-35.

8. Elgqvist J. Nanoparticles as theranostic vehicles in experimental and clinica applications-focus on prostate and breast cancer. Int J Mol Sci. 2017; 18: $1-53$.

9. Malik A, Tahir Butt T, Zahid S, Zahid F, Waquar S, Rasool M, et al. Use of Magnetic Nanoparticles as Targeted Therapy: Theranostic Approach to Trea and Diagnose Cancer. J Nanotechnol. 2017; 2017.

10. Abedin MR, Umapathi S, Mahendrakar $H$, Laemthong $T$, Coleman $H$ Muchangi D, et al. Polymer coated gold-ferric oxide superparamagnetic nanoparticles for theranostic applications. J Nanobiotechnology. 2018; 16: 1-13.

11. Baktash MS, Zarrabi A, Avazverdi E, Reis NM. Development and optimization of a new hybrid chitosan-grafted graphene oxide/magnetic nanoparticle system for theranostic applications. J Mol Liq. 2021; 322: 114515

12. Dong Q, Yang H, Wan C, Zheng D, Zhou Z, Xie S, et al. Her2-Functionalized Gold-Nanoshelled Magnetic Hybrid Nanoparticles: a Theranostic Agent for Dual-Modal Imaging and Photothermal Therapy of Breast Cancer. Nanoscale Res Lett. 2019; 14.

13. Gobbo OL, Sjaastad K, Radomski MW, Volkov Y, Prina-Mello A. Magnetic nanoparticles in cancer theranostics. Theranostics. 2015; 5: 1249-1263.

14. Semkina AS, Abakumov MA, Skorikov AS, Abakumova TO, Melnikov PA Grinenko NF, et al. Multimodal doxorubicin loaded magnetic nanoparticles for VEGF targeted theranostics of breast cancer. Nanomedicine Nanotechnology, Biol Med. 2018; 14: 1733-1742.

15. Nosrati $H$, Adibtabar $M$, Sharafi $A$, Danafar $H$, Hamidreza Kheiri $M$. PAMAM-modified citric acid-coated magnetic nanoparticles as $\mathrm{pH}$ sensitive biocompatible carrier against human breast cancer cells. Drug Dev Ind Pharm. 2018; 44: 1377-1384.

16. Chang $\mathrm{CH}$, Tsai IC, Chiang CJ, Chao YP. A theranostic approach to breast cancer by a quantum dots- and magnetic nanoparticles-conjugated peptide. J Taiwan Inst Chem Eng. 2019; 97: 88-95. 